\title{
Reflets
}

Revue d'intervention sociale et communautaire

\section{Les effets d'un partenariat entre l'Université de Saint-Boniface et les organismes communautaires dans la formation des intervenantes et intervenants à travers le Projet transdisciplinaire en santé communautaire (PTSC)}

\author{
Halimatou Ba, Florette Giasson, Hélène Archambault et Julie Gauvin
}

Volume 22, numéro 2, automne 2016

URI : https://id.erudit.org/iderudit/1038976ar

DOI : https://doi.org/10.7202/1038976ar

Aller au sommaire du numéro

Éditeur(s)

Reflets, Revue d'intervention sociale et communautaire

ISSN

1203-4576 (imprimé)

1712-8498 (numérique)

Découvrir la revue

Citer cet article

Ba, H., Giasson, F., Archambault, H. \& Gauvin, J. (2016). Les effets d'un partenariat entre l'Université de Saint-Boniface et les organismes communautaires dans la formation des intervenantes et intervenants à travers le Projet transdisciplinaire en santé communautaire (PTSC). Reflets, 22(2),

174-185. https://doi.org/10.7202/1038976ar
Résumé de l'article

En milieu minoritaire francophone, les organismes communautaires jouent un rôle important dans la prise en charge des populations vulnérables. Ils constituent un groupe particulièrement à risque à cause de la complexité de leurs besoins qui sont souvent liés aux déterminants sociaux de la santé. Cette prise en charge nécessite de la part des intervenantes et intervenants sociaux des compétences professionnelles transdisciplinaires et des pratiques renouvelées. Pour favoriser la pratique transdisciplinaire dans le domaine des services de santé et services sociaux en milieu minoritaire francophone et réduire les inégalités sociales que vivent les populations, l'Université de Saint-Boniface a lancé le Projet transdisciplinaire en santé communautaire, en partenariat avec des organismes qui oeuvrent dans la communauté francophone à Winnipeg. L'article décrit cette recherche-action, qui a été réalisée durant trois années consécutives, en concomitance avec la formation théorique. Les résultats permettent de décrire l'acquisition de compétences professionnelles par les étudiantes et étudiants inscrits au projet, l'intégration de l'approche transdisciplinaire dans les pratiques au sein des organismes communautaires, ainsi que le renforcement d'un partenariat université-organismes communautaires. L'article identifie quelques enjeux et recommandations issus de cette formation transdisciplinaire.
Tous droits réservés @ $\mathbb{0}$ Reflets, Revue d’intervention sociale et communautaire, 2017
Ce document est protégé par la loi sur le droit d'auteur. L'utilisation des services d’Érudit (y compris la reproduction) est assujettie à sa politique d'utilisation que vous pouvez consulter en ligne. 


\title{
Les effets d'un partenariat entre l'Université de Saint- Boniface et les organismes communautaires dans la formation des intervenantes et intervenants à travers le Projet transdisciplinaire en santé communautaire (PTSC)
}

\author{
Halimatou Ba, Ph.D. \\ Professeure agrégée, Université de Saint-Boniface \\ Florette Giasson, M. Serv. Soc. \\ Professeure, Université de Saint-Boniface \\ Hélène Archambault, Ph.D. \\ Professeure agrégée, Université de Saint-Boniface \\ Julie Gauvin, M. Sc. \\ Assistante de recherche, Université de Saint-Boniface
}

\section{Résumé}

En milieu minoritaire francophone, les organismes communautaires jouent un rôle important dans la prise en charge des populations vulnérables. Ils constituent un groupe particulièrement à risque à cause de la complexité de leurs besoins qui sont souvent liés aux déterminants sociaux de la santé. Cette prise en charge nécessite de la part des intervenantes et intervenants sociaux des compétences professionnelles transdisciplinaires et des pratiques renouvelées. Pour favoriser la pratique transdisciplinaire dans le domaine des services de santé et services sociaux en milieu minoritaire francophone et réduire les inégalités sociales que vivent les populations, l'Université de Saint-Boniface a lancé le Projet transdisciplinaire en santé communautaire, en partenariat avec des organismes qui œuvrent dans la communauté francophone à Winnipeg. L'article décrit cette rechercheaction, qui a été réalisée durant trois années consécutives, en concomitance avec la formation théorique. Les résultats permettent de décrire l'acquisition de compétences 
professionnelles par les étudiantes et étudiants inscrits au projet, l'intégration de l'approche transdisciplinaire dans les pratiques au sein des organismes communautaires, ainsi que le renforcement d'un partenariat université-organismes communautaires. L'article identifie quelques enjeux et recommandations issus de cette formation transdisciplinaire.

\section{Introduction}

Il existe actuellement une tendance à encourager les professionnelles et professionnels issus de différentes disciplines à travailler en équipe et à pratiquer diverses formes de partenariat et de collaboration (Létourneau, 2008). En effet, les pratiques disciplinaires en solo, qui comportent des langages spécialisés et spécifiques, sont de moins en moins considérées (Létourneau, 2008). Dans le domaine des services de santé et services sociaux, les problèmes sociaux sont si complexes que leur résolution nécessite souvent l'éclairage de plusieurs disciplines. Cette situation impose aux futurs intervenantes et intervenants professionnels non seulement la maittrise de leur propre domaine d'intervention, mais aussi la prise en compte de la contribution d'autres disciplines dans la prise en charge de ces mêmes problèmes (Lafargue, 2007). Pour ce faire, au cours de leur formation, ces professionnelles et professionnels gagneraient à être initiés à la démarche transdisciplinaire, qui considère l'apport de plusieurs disciplines à l'édification d'un cadre qui englobe et dépasse ce que chaque discipline connaît partiellement (Létourneau, 2008; Chartier, 2012). Ainsi, ils pourront démontrer leur capacité de devenir des expertes et experts de cette démarche transdisciplinaire.

C'est pour permettre ce dépassement disciplinaire que l'Université de SaintBoniface (USB), en collaboration avec des organismes communautaires, a lancé le projet transdisciplinaire en santé communautaire (PTSC), grâce au financement reçu de la compagnie d'assurance, la Great-West Life (Archambault, et collab., 2014). S’inspirant d'autres programmes transdisciplinaires existant déjà dans d'autres universités canadiennes (Charbonneau, 2009), ce projet avait pour but de créer un partenariat qui servirait de passerelle entre l'USB et la communauté par le biais de la formation. Il s'agissait aussi de mieux préparer les ressources humaines francophones à travailler en équipe transdisciplinaire et à fournir des services en langue française dans la communauté tout en répondant aux différents besoins des populations en matière de santé et de services sociaux.

D'une part, la recherche-action entreprise dans le cadre de ce projet visait à offrir aux étudiantes et étudiants des occasions d'apprentissage collaboratif, coopératif et expérientiel 
dans une équipe transdisciplinaire afin de permettre l'acquisition de compétences transdisciplinaires au cours de la formation et de favoriser l'intégration durable de ces compétences dans leurs pratiques professionnelles après la formation. D'autre part, il s'agissait d'offrir de l'aide aux organismes communautaires en leur permettant de développer des potentialités visant l'innovation dans leurs interventions.

Le projet consistait à offrir, en partenariat avec des organismes communautaires, une formation théorique et pratique à des étudiantes et étudiants inscrits et issus de plusieurs disciplines enseignées à l'USB. Les étudiantes et étudiants inscrits, réunis en équipes transdisciplinaires, devaient suivre ensemble des cours théoriques en classe. En même temps, ils devaient réaliser des activités pratiques dans la communauté auprès des organismes communautaires impliqués dans le projet. Tout en réalisant ces activités en équipes, ces étudiantes et étudiants devaient développer des compétences transdisciplinaires. À la fin de chaque année, chaque équipe devait rédiger un rapport décrivant l'expérience réalisée auprès de l'organisme communautaire et faire une présentation publique de ses travaux au moyen d'affiches que les membres de l'équipe avaient eux-mêmes confectionnées. Tous les étudiants et étudiantes inscrits devaient finalement participer à des entrevues visant à recueillir leurs opinions sur le déroulement et l'impact du projet.

\section{Cadre théorique et méthodologique}

La recherche-action (Patton, 2011) de ce projet a été abordée selon une approche transdisciplinaire et s'est effectuée en même temps que se déroulait la formation théorique. De l'avis de plusieurs auteurs (Morin, 1999; Bauzà, 2012; Claverie, 2010; Paquot, 2013), la transdisciplinarité va au-delà de toute discipline et les traverse toutes. Considérée comme un système total sans frontières stables entre les disciplines, la transdisciplinarité réfere à une approche où l'atteinte d'un consensus et l'ouverture des limites professionnelles sont essentielles. Avec l'approche transdisciplinaire, il est attendu que les étudiantes et étudiants travaillent ensemble pour atteindre un consensus leur permettant d'obtenir un résultat qui dépasse leurs limites professionnelles.

La recherche-action a débuté en janvier 2013 auprès d'une première cohorte d'étudiantes et étudiants. Elle s'est poursuivie auprès d'une deuxième cohorte en 2014, puis d'une troisième en 2015. La population à l'étude est composée des étudiantes et étudiants inscrits pendant la durée du projet et des personnes-ressources qui les encadraient dans les organismes communautaires partenaires. Quatorze étudiantes et étudiants étaient 
impliqués, dont huit provenant des sciences infirmières, cinq du service social et un de la psychologie. Onze personnes-ressources, représentant les cinq organismes partenaires, étaient disponibles pour les encadrer. Une professeure de l'équipe de recherche avait pour mission d'observer les étudiantes et étudiants deux fois par semestre, au début et à la fin de leur expérience pratique, pour identifier les compétences transdisciplinaires qu’ils développaient lorsqu'ils travaillaient en groupe. Les données de cette observation ont été recueillies au moyen d'un instrument intitulé « Rubrique d'évaluation de la collaboration interpersonnelle ${ }^{1} »$. Cet instrument de mesure permet d'évaluer six compétences transdisciplinaires, soit la communication, la collaboration, les rôles et responsabilités, l'approche en collaboration avec l'organisme centrée sur la clientèle, le fonctionnement de l'équipe, et la gestion ou résolution de conflits. Pour compléter la constitution des données, à la fin de chacune des trois années, des entrevues ont été réalisées auprès des groupes d'étudiantes et étudiants et auprès des personnes-ressources afin d'évaluer l'impact de l'expérience pratique sur les étudiantes et étudiants et l'impact du projet sur le fonctionnement des organismes. Les données recueillies ont été transcrites, analysées et codifiées selon la méthode décrite par L'Écuyer (1990).

\section{Résultats}

Dans cette partie seront évoqués quelques résultats de cette recherche-action, qui spécifieront l'impact du projet sur les organismes communautaires, les compétences transdisciplinaires développées par les étudiantes et étudiants, et la perception que les étudiantes et étudiants ont du projet. Quelques suggestions et recommandations seront émises en guise de conclusion.

\section{Impact du projet sur les organismes communautaires}

Cinq organismes communautaires francophones à Winnipeg ont participé à la réalisation du PTSC. Ces organismes s'intéressent respectivement aux questions de santé et de bienêtre communautaire, aux services de santé primaires, à l'élaboration de projets pour les aînés francophones, à la distribution de biens matériels et à l'accueil des immigrantes et immigrants francophones. Lors des collaborations avec ces organismes, les étudiantes et étudiants du PTSC ont réalisé des expériences pratiques proposées par ces organismes en fonction de leurs besoins respectifs. C'est ainsi que les étudiantes et étudiants ont créé un répertoire détaillé des logements abordables dans une zone géographique de Winnipeg. Ils ont effectué des évaluations des besoins non matériels des personnes démunies et de 
l'état des services en santé mentale offerts aux jeunes francophones. Ils ont complété la mise sur pied d'un service alimentaire et ont aidé au dépistage des besoins des aînés francophones et des aînés immigrants à Saint-Boniface. Enfin, ils ont créé et mis sur pied un groupe de soutien pour briser l'isolement et favoriser l'intégration sociale des immigrantes et immigrants âgés de 65 ans et plus.

Les organismes communautaires impliqués dans le projet ont bien apprécié leur partenariat avec l'Université de Saint-Boniface. D'ailleurs, plusieurs d'entre eux collaboraient déjà avec l'Université dans le cadre de la réalisation de stages. Les personnesressources ont estimé que le temps alloué à la réalisation du projet communautaire était assez court et ne favorisait pas la compréhension théorique de la transdisciplinarité au début du partenariat, ce qui a eu pour effet de ralentir la progression de l'exécution des expériences sur le terrain. Selon les personnes-ressources, les étudiantes et étudiants ont bien répondu aux besoins des organismes en réalisant des travaux qui n'auraient sans doute pas pu être faits sans eux. Leur présence a pu combler un grand besoin de personnel supplémentaire, et les rapports qu'ils ont produits constituent des outils importants pour les organismes.

"Ça a ouvert les yeux, quelle contribution les étudiants ont faite! Ça a donné une perspective beaucoup plus large que nous on avait ciblée. " "Je dirais qu'elles ont même dépassé [les objectifs]. » (AF2015)

"We were always about dealing with the needs of the vulnerable and the unmet needs of those populations specifically. So this was one area where we managed to, with the help of your students, truly answer an unmet need. So that philosophically felt really good to see that that is actually helping address something. " (CY2013)

D’autres avantages ont été constatés, par exemple le fait de bénéficier d'une expertise externe permettant de soulever de nouveaux enjeux bénéfiques pour les organismes et leur clientèle et de donner plus de poids aux dossiers que les organismes pourraient présenter à leur conseil d'administration ou aux investisseurs.

" Ça emmène une certaine crédibilité [au projet] parce que c'est une tierce partie qui fait la recherche. »(Cat2014)

Le projet a permis de renforcer les liens de partenariat entre l'Université et la communauté. 
"C'était vraiment la première fois que je voyais la communauté être avec l'université qui est dans notre milieu. »(Cat2014)

« [...] c'est un heureux partenariat que nous [souhaitons] maintenir et développer davantage. Et c'est bien perçu par moi, et [...] tant au niveau de notre conseil d'administration qu'au niveau corporatif. " (FL2013)

En plus, les organismes ont pu bénéficier d'une main-d'œuvre supplémentaire pour accomplir des projets importants pour leur clientèle cible respective. De ces points de vue, l'importance de maintenir le PTSC n'est plus à démontrer.

Les étudiantes et étudiants qui ont pris part à ce projet ont été répartis en sept équipes de deux pour travailler à la réalisation de sept mini-projets proposés par les organismes communautaires. Cependant, il a été suggéré que tous les étudiants et étudiantes du cours auraient pu travailler ensemble sur un même grand projet plutôt que sur des projets en petits groupes auprès de différents organismes.

" [Il faut que] toute l'équipe qui travaille ensemble pour faire quelque chose de vrai et d'avoir toutes les étapes et de [...] faire une recherche approfondie et [...] une intervention approfondie. [...] Si on veut vraiment faire un impact sur la communauté, et je pense que ça c'est le but de ce cours, we need to make it bigger. » (FL2014)

Or, le PTSC a eu un impact très notoire sur la communauté grâce à la participation effective et positive de cinq organismes. La volonté de maintenir les partenariats avec tous les organismes partenaires a donc nécessité la création des sept équipes pour la mise en œuvre de tous les projets proposés. Cependant, l'idée de se concentrer sur un seul projet par cohorte est très pertinente, compte tenu de l'effectif très réduit d'étudiantes et étudiants et du nombre de disciplines visées par le projet. Le regroupement de tous les étudiants et étudiantes de chaque cohorte autour d'un seul projet aurait certainement eu un impact plus grand dans la communauté et aurait permis de faire travailler tous les étudiants et étudiantes ensemble pour mieux faire ressortir les compétences transdisciplinaires recherchées.

\section{Les compétences transdisciplinaires développées par les étudiantes et étudiants}

Afin de déterminer si les étudiantes et étudiants ont développé des compétences transdisciplinaires, on les a suivis sur la base de la « Rubrique d'évaluation de la 
collaboration interprofessionnelle ", conçue pour évaluer le travail en équipe dans le milieu de la santé. Tous les étudiants et étudiantes ont été évalués à quatre reprises tout au long de leur participation au projet. Les données montrent clairement une acquisition de compétences au fur et à mesure de l'avancement du projet pour chacune des trois cohortes. La compilation de ces résultats donne le tableau suivant:

\section{Tableau 1}

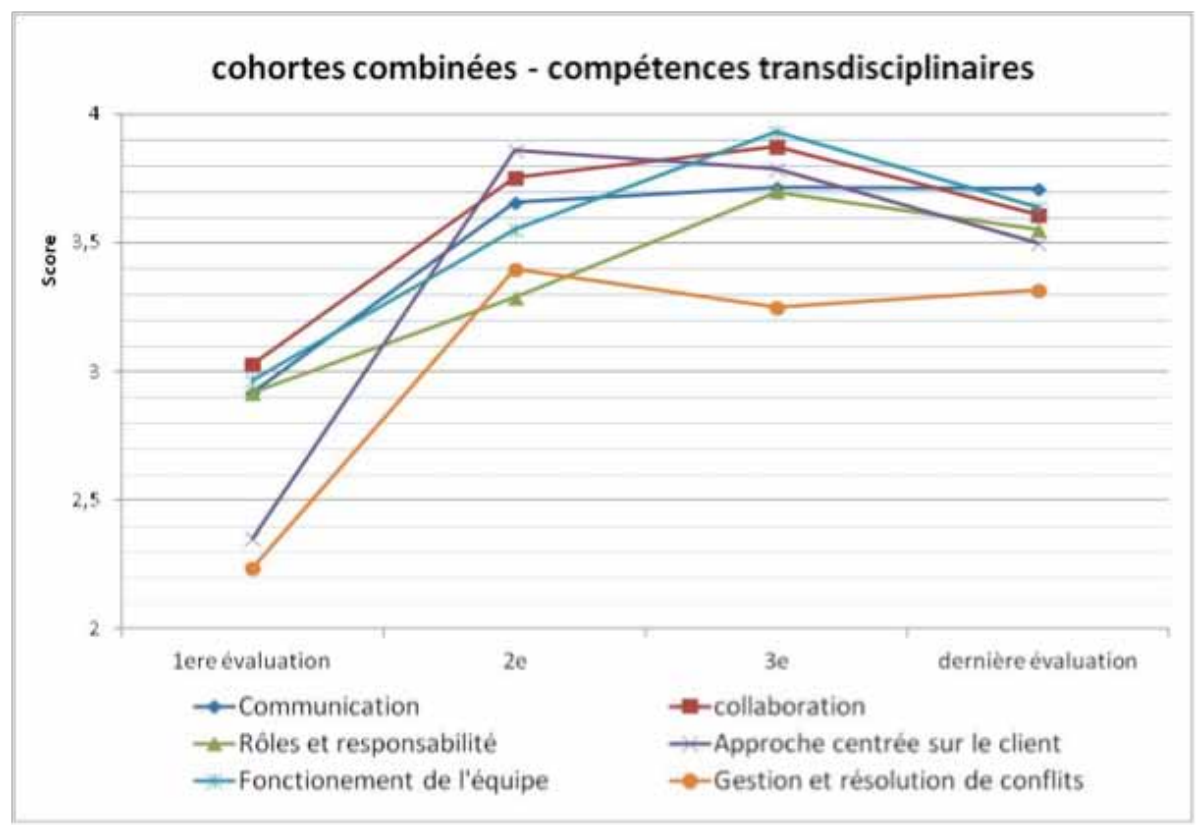

La première compétence mesurée est la communication. Les étudiantes et étudiants ont été observés relativement à leur capacité à communiquer de manière respectueuse en employant des stratégies de facilitation. Selon les données qualitatives recueillies, ils ont su partager leurs connaissances personnelles et faire preuve d'écoute et d'intérêt à l'égard des propos de leur partenaire. Cependant, malgré ces points positifs, les mêmes données qualitatives indiquent que certains étudiants et étudiantes avaient de la difficulté à exprimer leurs opinions. Au sujet de la collaboration entre membres du groupe, une amélioration a été observée en ce qui concerne l'établissement de relations, l'intégration des opinions dans la prise de décision et l'obtention du consentement des individus 
concernés. Ces compétences se sont développées et maintenues pendant toute la durée de l'expérience communautaire. Quant à l'échange d'informations avec les autres professionnelles et professionnels, il a augmenté pour atteindre son maximum à la fin du projet. Par rapport à leurs rôles et responsabilités, les étudiantes et étudiants ont pu les décrire auprès des autres membres de l'équipe et intégrer ceux des coéquipiers aux solutions élaborées, ce qui a permis d'optimiser le résultat de l'intervention. Cependant, certains étudiants et étudiantes avaient plus de difficulté à maintenir leurs responsabilités au sein de l'équipe et à expliquer clairement leur pratique personnelle.

En matière de collaboration centrée sur la clientèle, la revendication de la participation du client et le partage d'informations avec ce dernier sont deux aspects qui se sont nettement améliorés vers la fin du projet. Cette compétence a été difficilement observée lorsque le temps de réalisation du projet a été réduit pour la troisième cohorte, ce qui souligne l'importance que les projets se déroulent sur deux sessions. Au sujet du fonctionnement de l'équipe, plusieurs aspects se sont améliorés avec le temps, notamment la participation aux discussions et les habiletés de travail en équipe. Le partage de leadership a causé le plus de difficultés aux équipes et n'a pas été facile dans tous les groupes. Enfin, pour toutes les cohortes, les habiletés de gestion et de résolution des conflits se sont nettement améliorées. À la fin du projet, les étudiantes et étudiants tenaient davantage compte de l'opinion des autres membres du groupe, faisaient preuve d'écoute et formulaient des demandes de précisions sur les différents points de vue de manière respectueuse.

\section{La perception du projet par les étudiantes et étudiants}

Les résultats obtenus ont permis d'avancer que les étudiantes et étudiants ont apprécié le cours « Projet transdisciplinaire en santé communautaire » et l'ont trouvé très exigeant et bénéfique. Ils estimaient que c'était une bonne expérience communautaire qui les préparait à la vie professionnelle puisqu'ils ont indiqué :

«En allant sur le terrain aussi on a rencontré plusieurs personnes des diverses disciplines. » (CS2013)

De plus, ils ont trouvé que la production de rapports de fin d'année et la confection d'affiches pour les présentations finales étaient enrichissantes. Ils ont perçu l'importance de pouvoir «mettre certains concepts [théoriques] en application et d'entrer en contact avec la population " (CS2014). Certains étudiants et étudiantes ont aimé voir comment des personnes de différents domaines perçoivent un même concept et parviennent à collaborer avec les membres des autres disciplines en présence. 
«[...] J'ai beaucoup aimé cette expérience-là. Surtout les symbioses, et puis la complexité de la santé et le tout qui vise un même objectif qui est le bienêtre; jrai beaucoup aimé ça. » (CS2013)

Le projet était basé sur une alternance entre les cours théoriques en classe et l'expérience communautaire sur le terrain. Cette formule partagée, qui semblait un peu redondante pour ceux et celles qui avaient plus d'expérience et plus de familiarité avec certains concepts, a été perçue comme essentielle pour les étudiantes et étudiants en début de formation.

«Il y avait plusieurs aspects du cours qui étaient pertinents à notre expérience communautaire. » (FL2013)

«[...] Tout ce qui a été dit durant le cours, c`est tout ce qu^on a pu appliquer sur le terrain lors de notre expérience communautaire. » (CY2015)

Le cours était offert par une équipe de professeurs et professeures venant de différents domaines. Les étudiantes et étudiants ont aimé avoir les différentes perspectives de chacune des professions, connaître différents styles d'enseignement et avoir accès à l'appui de plus de professeurs et professeures.

"Moi j'ai aimé parce que tu as le point de vue et l'approche de plusieurs différents professeurs. » (AF2014)

Ils ont pu observer les interactions entre les enseignantes et enseignants qui appliquent ces aspects de collaboration à leurs propres projets et ils ont affirmé :

"C'était un bon exemple de travail d'équipe. » (FL2013)

"Ça nous a aidés un peu à, comme, être plus flexibles et plus ouverts à des changements. » (AF2015)

Le cours s'étalait sur deux semestres lors des deux premières années. En automne, il était axé sur les aspects théoriques en classe, la préparation des équipes et la sélection des projets soumis par les organismes. L'exécution et la finalisation des projets se déroulaient en hiver. Cette formule a été bien appréciée par certains étudiants et étudiantes, comme en témoigne l'un d'entre eux :

"Je pense que ce cours a vraiment besoin de deux semestres parce que crest un cours intense, et puis avec beaucoup d`expérience sur le terrain. » (CY2013) 
Cependant, pour la dernière cohorte, l'équipe a dû apporter des changements dans l'organisation du cours et condenser tout le contenu en automne. Ce remaniement est intervenu à la suite des commentaires formulés par les étudiantes et étudiants lors des entrevues précédentes. D’une part, ils ont émis des critiques concernant l'équilibre des tâches entre les deux sessions. Ils sont beaucoup plus actifs et créatifs en hiver lorsqu'ils sont sur le terrain que lorsqu'ils suivent de la théorie en classe. D'autre part, ils se sont prononcés sur le nombre de crédits alloués au cours :

"J'aurais voulu peut-être que ça soit plus comme un cours de six crédits [commencé] sur le terrain dès le mois de septembre. Comme ça, on pourrait vraiment faire plus un grand impact. Parce que ce n'était vraiment juste pas assez de temps, puis je pense qu'on aurait vraiment pu faire plus de choses si on avait eu plus de temps. » (FL2014)

En effet, pour les étudiantes et étudiants, avec une valeur de six crédits au lieu de trois, le cours pourrait être plus attrayant et refléterait avec plus de justesse le nombre d'heures consacrées au projet en classe et sur le terrain. Ainsi, ils pourraient consacrer encore plus de temps à l'expérience communautaire qu'ils ont tous bien appréciée. Leurs rencontres avec les organismes ont été enrichissantes pour la présentation, la préparation et la mise à exécution des projets. La majorité des décisions ont été prises par les étudiantes et étudiants qui se sont sentis soutenus par des personnes-ressources très accommodantes dans leur accompagnement.

\section{Conclusion et recommandations}

Mettre en place un partenariat entre l'Université de Saint-Boniface et des organismes communautaires travaillant auprès des populations francophones à Winnipeg a été une expérience d'apprentissage très enrichissante. Les interventions ont apporté des éclairages sur quelques inégalités sociales et problèmes sociaux qui constituent des enjeux pour les organismes communautaires partenaires. À la suite des trois années du projet pilote, quelques recommandations ont été formulées pour améliorer l'expérience de cette pratique transdisciplinaire.

Du côté des organismes partenaires, l'idéal serait de présenter aux étudiantes et étudiants des projets plus pratiques qui les amèneraient directement sur le terrain. En effet, quelques projets présentés par eux sont des projets de recherche au lieu d'être des projets d'intervention. Pour éviter ce genre de situations tout en respectant les besoins 
des organismes partenaires, il faut consacrer un temps suffisant au travail préparatoire pour permettre à chaque partie de mieux comprendre ses rôles et responsabilités dans le partenariat et les buts poursuivis par le PTSC. Du côté de l'Université, il est essentiel de faire la promotion du cours pour favoriser un recrutement massif d'étudiantes et étudiants de plusieurs disciplines afin de maximiser l'aspect transdisciplinaire du projet. Le cours doit avoir une valeur de six crédits et se dérouler sur deux semestres, avec un équilibre entre la théorie et la pratique. Aussi, l'expérience communautaire devrait démarrer plus tôt dans l'année, soit en même temps que les cours théoriques. Enfin, les étudiantes et étudiants visés devraient être suffisamment avancés dans leurs programmes respectifs et être plus à l'aise avec leurs propres professions afin d'être mieux en mesure de transcender leurs propres perspectives et d'agir de manière transdisciplinaire avec les autres professions.

\section{Note}

Faculté de médecine, Memorial University of Newfoundland, 2010.

\section{Bibliographie}

ARCHAMBAULT, Hélène, et collab. (2014). «Limplantation du projet transdisciplinaire à l'Université de Saint-Boniface ", Reflets: revue d'intervention sociale et communautaire, Vol. 20, № 1, p. 108-119.

BAUZÀ, Hugo Francisco (2012). "La transdisciplinarité », Caietele Echinox, Vol. 22, p. 342-352, http://www.ceeol.com/search/article-detail?id=48089

CHARBONNEAU, Léo (2009). " Des liens avec la communauté. Les étudiants, artisans de la popularité grandissante de projets d'apprentissage par le service communautaire dans l'ensemble du pays. ", Affaires universitaires, réf. du 8 novembre 2009, http://www.affairesuniversitaires.ca/ articles-de-fond/article/des-liens-avec-la-communaute/

CHARTIER, Jean-Pierre (2012). «Dela pluridisciplinaritéà la transdisciplinarité», Cliniques, № 1, p. 96-114, http://www.cairn.info.uml.idm.oclc.org/revue-cliniques-2012-1-page-96.htm

CLAVERIE, Bernard (2010). «Pluri-, inter-, transdisciplinarité : ou le réel décomposé en réseaux de savoir ", Projectics / Proyéctica / Projectique, No 1, p. 5-27, http://www.cairn.info.uml.idm.oclc.org/revue-projectique-2010-1-page-5.htm

CONSEIL ACADÉMIQUE EN SANTÉ (2009). Rubrique d'évaluation de la collaboration interprofessionnelle. 
LAFARGUE, M. (2007). "Démarche Systèmes et Transdisciplinarité dans la Formation des Ingénieurs ", European Journal of Engineering Education, Vol. 13, № 3, p. 271-281, http://dx.doi.org/10.1080/03043798808939426

LÉTOURNEAU, Alain (2008). "La transdisciplinarité considérée en général et en sciences de l'environnement ", [VertigO] La revue électronique en sciences de l'environnement, Vol. 8, $\mathrm{N}^{\circ} 2$, p. 1-9, http://id.erudit.org/iderudit/019961ar

L'ÉCUYER, René (1990). Méthodologie de l'analyse développementale de contenu : méthode GPD et concept de soi, Sillery, Presse de l'Université du Québec, 502 p.

MORIN, Edgar (1999). Les sept savoirs nécessaires à l'éducation du futur, Paris, Éditions du Seuil, $129 \mathrm{p}$.

PAQUOT, Thiery (2013). "Édition : délicate pratique de la transdisciplinarité ", Hermès, La Revue, $\mathrm{N}^{\circ}$ 3, p. 145-146, http://www.cairn.info.uml.idm.oclc.org/article_p.php?ID_ARTC

PATTON, Michael Quinn (2011). Developmental Evaluation:Applying Complexity Concepts to Enhance Innovation and Use, New York, The Guilford Press, 375 p. 